\title{
LOS DATOS EN TWITTER COMO FUENTE DEL DISCURSO ORAL COLOQUIAL: ESTUDIO DE CASO DEL MARCADOR DISCURSIVO EN PLAN
}

\author{
Emma DE SMET \\ Universidad de Gante (Bélgica) \\ Renata ENGHELS \\ Universidad de Gante (Bélgica)
}

\section{RESUMEN}

En las últimas décadas, se ha observado un interés creciente por fenómenos lingüísticos típicos del español oral conversacional. Sin embargo, tal expansión del campo de investigación contrasta fuertemente con la disponibilidad restringida de datos empíricos orales. Como reacción, los investigadores han recurrido a bancos de datos alternativos, como los medios sociales. El presente artículo contribuye a la pregunta de si el lenguaje usado en Twitter se puede considerar una fuente sólida para estudiar rasgos del español coloquial. En concreto, compara el uso en Twitter de uno de los marcadores discursivos más difundidos en el español contemporáneo (entre jóvenes), a saber, en plan, con su uso en corpus orales espontáneos (como COLAm). Los resultados muestran que, por un lado, los hablantes adoptan un estilo expresivo y subjetivo similar al que se observa en corpus orales conversacionales; por otro, debido a su carácter monológico y asincrónico, Twitter parece ser menos apropiado para estudiar fenómenos relacionados con la estructura dialógica típica de datos espontáneos, incluso las funciones metadiscursivas inherentes a los cambios de turnos.

PALABRAS CLAVE: español coloquial, ciberlenguaje, Twitter, marcadores discursivos, en plan.

\section{AbSTRACT}

The last decades have witnessed a growing interest in linguistic phenomena typical of spoken conversational Spanish. However, this expansion of the research field contrasts sharply with the rather limited availability of empirical oral data. As a reaction, researchers have increasingly turned to alternative databases, such as social media. This article particularly looks into the question to what extent the language used on Twitter can count as a reliable source of colloquial Spanish. In particular, it compares the profile of one of the most frequently used discourse 
markers in contemporary colloquial (youth) Spanish, namely en plan, on Twitter with data taken from spoken corpora (such as COLAm). The results indicate that, on the one hand, language users do turn to an expressive and subjective discourse style on Twitter, similar to how it would be found in an oral conversational corpus. On the other hand, due to its monological and asynchronous character, Twitter appears to be less suited to study phenomena related to the conversational change-of-turns structure of spontaneous data, including mostly metadiscourse functions.

KEYWORDS: colloquial Spanish, cyber language, Twitter, discourse markers, en plan.

Fecha de recepción: 22/10/2019

Fecha de aceptación: 11/12/2019

Fecha de la versión definitiva: 14/01/2020

Another beautiful day for art to imitate life.

Life to imitate art. And Twitter to imitate neither.

(-@NeinQuarterly, 15/07/2019)

\section{INTRODUCCIÓN: TWITTER COMO CORPUS LINGÜÍSTICO}

A partir de los años setenta, se ha producido en la investigación lingüística un «giro oralista» (López Serena y Borreguero Zuloaga 2010: 415), es decir, una reorientación teórico-metodológica por la que han pasado a ocupar un lugar más destacado fenómenos discursivo-pragmáticos propios del registro oral coloquial, como los marcadores discursivos (de aquí en adelante MMDD) (Fuentes 1996; Portolés 1998; Zorraquino y Portolés 1999). Para investigar estos fenómenos, es necesario poder contar con datos típicos del lenguaje hablado coloquial, recogidos en corpus orales (Recalde y Vázquez Rosas 2009).

No obstante, autores como Gilquin y De Cock (2011) han llamado la atención sobre las limitaciones que conlleva la investigación lingüística a base de corpus orales. En primer lugar, muchas veces se carece de datos recientes y actualizados (Briz 2012). Este problema se hace evidente si tomamos en cuenta los corpus orales en el mundo hispánico: los datos más recientes del CORPES XXI (el corpus actual de la RAE), por ejemplo, provienen del año 2016. Segundo, los corpus orales a menudo se basan en entrevistas (semi-) dirigidas que, como es bien sabido, constituyen una situación discursiva particular, con propios fines comunicativos. Los papeles tanto del entrevistador como del entrevistado son más predeterminados y menos anclados en la vida real. Además, en general, los participantes son desconocidos y tienen una relación más distante. Por consiguiente, suscitan una forma de hablar más premeditada (que corresponde más a usos 
y funciones convencionales y menos expresivas), no-conversacional y más formal. Para evitar estos problemas, se han creado corpus conversacionales basados en datos espontáneos, pero se ha cuestionado la autenticidad de estos datos también (Shanmuganathan 2005). Ya que no se permiten las grabaciones secretas, datos completamente espontáneos y coloquiales son difíciles de obtener. Los informantes siempre serán conscientes de que son observados y grabados, y por eso adaptarán su habla (se trata de la llamada «paradoja del observador»).

Para hacer frente a estos desafíos, se han buscado otras fuentes que reflejen el uso oral y espontáneo del lenguaje, entre otras, las redes sociales y los medios de comunicación como Twitter. En el ámbito de la lingüística hispánica, por ejemplo, se observa que los autores recurren cada vez más a datos recopilados en Twitter para analizar diferentes fenómenos lingüísticos, como por ejemplo MMDD (Pano Alamán y Mancera Rueda 2014; Padilla Herrada 2016) y neologismos (González Fernández 2017). Para estos autores, las ventajas ofrecidas por un corpus elaborado en Twitter son, entre otras, la libertad y espontaneidad con la que hablan los usuarios y por lo tanto la autenticidad de los datos, su carácter actualizado y el acceso a datos geolocalizados.

Sin embargo, se plantea de manera cada vez más acuciante la pregunta de si la variante lingüística utilizada en Internet (el llamado ciberlenguaje; Crystal 2001; Herring 1996, 2004; Overbeck 2017; Yus 2001, 2011) puede considerarse como una imagen fiable del lenguaje hablado y coloquial. Por una parte, por su alto grado de espontaneidad, su fin interpersonal y la relación de igualdad entre los hablantes (Briz 1998, 2003), la variante ha sido definida como un conjunto de «textos escritos oralizados» (Yus 2001) o «conversaciones escritas» (Pano Alamán y Mancera Rueda 2014). Por otra parte, hay quienes dicen que la variante difiere bastante de la conversación oral coloquial y manifiesta ante todo características de la lengua escrita: existe entre los hablantes una distancia locativo-temporal (Nishimura 2013), y los intercambios se suceden linealmente de forma asincrónica a través de un código gráfico (Padilla Herrada 2016).

Como consecuencia, el ciberlenguaje problematiza la clasificación binaria de los tipos de lenguaje entre escrito y oral basada en el medio a través del cual se produce un texto (que puede ser gráfico o fónico). Se necesita entonces una clasificación más matizada basada en la manera en que está concebido el texto (que puede ser hablado o escrito) ${ }^{1}$. Más en concreto, la concepción de un texto depende, según Koch y Oesterreicher (2007), de

\footnotetext{
${ }^{1}$ Un discurso político, por ejemplo, es medialmente fónico pero se concibe como escrito, mientras que una carta a un amigo es medialmente gráfico pero se concibe como hablado y más coloquial (Biber 1988).
} 
su «distancia comunicativa», que se define como la distancia (tanto física como social) entre los hablantes en un discurso. Esta distancia comunicativa depende, a su vez, de varios factores: la familiaridad entre los hablantes, la privacidad del discurso, la espontaneidad, etc. Con base en estos parámetros se ha desarrollado el continuum de la distancia comunicativa, ilustrado por la Figura 1. Los polos extremos están ocupados por el lenguaje coloquial oral por un lado, caracterizado por la inmediatez comunicativa y su concepción hablada, y el lenguaje formal escrito por otro lado, caracterizado por la distancia comunicativa y la concepción escrita.

Figura 1. Continuum de la distancia comunicativa (basado en Koch y Oesterreicher 2007: 34)

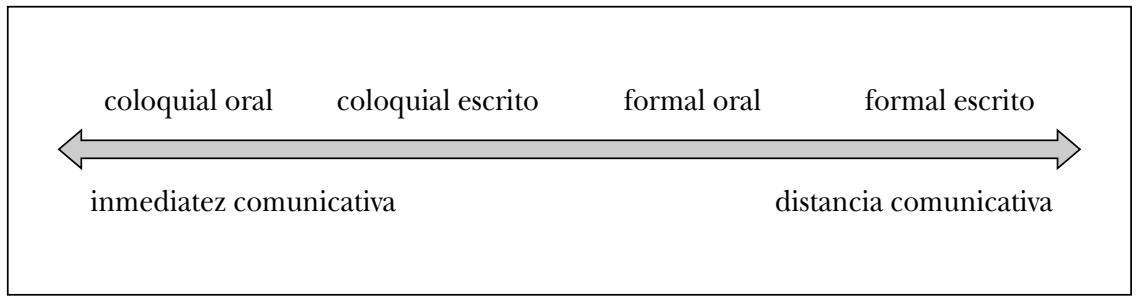

El presente estudio contribuye a la discusión sobre el lugar que ocupa el lenguaje utilizado en Twitter en este continuum y analiza en qué medida esta variante se acerca al registro oral coloquial. De hecho, varios autores afirman que la variante se sitúa en el plano de la inmediatez comunicativa (Yus 2001; Briz 2014; Pano Alamán y Mancera Rueda 2014; Pano Alamán 2015), pero raras veces se presentan datos que confirmen esta hipótesis.

La presente investigación se basa en un estudio de caso concreto, a saber el uso de la locución en plan como MD en el español peninsular, una de las expresiones lingüísticas que más ha llamado la atención en los últimos años. Siendo originariamente una locución adverbial, a partir de los años setenta el elemento poco a poco ha pasado a cumplir la función de MD por un proceso de gramaticalización ${ }^{2}$. La selección de en plan como estudio de caso se justifica por diferentes razones. Primero, el estudio de los MMDD en general resulta útil en el marco de la investigación sobre las diferencias entre la lengua escrita y hablada, por lo que muchos autores (Gili Gaya 1943; Koch y Oesterreicher 1990; Zorraquino y Portolés 1999) ya se han ocupado de la distribución y función de estos elementos en los

${ }^{2}$ Para una descripción detallada de este proceso de gramaticalización, véase Rodríguez Abruñeiras (2015) y De Smet (2019). 
dos registros (Enghels y Azofra Sierra 2018). A modo de ilustración, López Serena y Borreguero Zuloaga (2010: 446) afirman que «[E]l tipo de funciones que desempeñan [...] los MMDD está en relación con el perfil conceptual más o menos inmediato o distante de discursos». Además, el propio elemento en plan es omnipresente en datos contemporáneos. Pons Rodríguez (2018, en línea) afirma que «en plan es el nuevo chicle, es flexible y está todo el día en la boca de nuestros hablantes más jóvenes». Por último, disponemos de los resultados de estudios previos sobre el comportamiento lingüístico del MD en el lenguaje oral coloquial (Nord 2006; Jørgensen 2009; Rodríguez Abruñeiras 2015), más específicamente en el corpus COLAm (Corpus Oral de Lenguaje Adolescente de Madrid). Por consiguiente, resulta interesante la comparación de los resultados de estos estudios con los resultados del análisis a base de Twitter, con el objetivo de comprobar si Twitter puede utilizarse para el estudio de la comunicación oral coloquial como alternativa a los corpus orales.

Más en concreto, el presente estudio quiere responder a las siguientes preguntas de investigación:

1) ¿Cuáles son las funciones discursivo-pragmáticas que asume el MD en plan hoy día en la red social Twitter?

2) ¿Hasta qué punto las funciones de en plan en Twitter se comparan con las que asume el MD en el lenguaje hablado coloquial?

3) ¿El lenguaje utilizado en Twitter puede considerarse como un reflejo del registro oral y coloquial?

Para responder a estas preguntas, el estudio se compone de cuatro partes principales después de esta introducción. En el apartado 1 se ofrece una descripción de las funciones discursivo-pragmáticas que asume en plan en el lenguaje hablado; se basa en los estudios previos sobre el MD en los corpus orales COLAm y CREA (Nord 2006; Jørgensen 2009; Rodríguez Abruñeiras 2015) y un corpus basado en archivos de audio en WhatsApp ${ }^{3}$ (Méndez Orense 2016). La sección 2 discute las funciones de en plan tal y como se observan en nuestro corpus de tuits. En la sección 3 se compara el uso de en plan en el lenguaje hablado coloquial con su uso en Twitter, con el objetivo de verificar si los usos de este marcador pueden indicarnos una tendencia por parte de los hablantes a adoptar el registro coloquial en Twitter. Concluimos el estudio en el apartado 4.

\footnotetext{
${ }^{3}$ Méndez Orense (2016) analiza en WhatsApp tanto mensajes de texto como archivos de audio. Puesto que nuestro objetivo es comparar las funciones de en plan en Twitter con las que asume en el lenguaje hablado, solo tomamos en cuenta las funciones encontradas en los archivos de audio.
} 
1. EN PLAN EN EL LENGUAJE HABLADO COLOQUIAL

Originariamente, en plan era un sintagma preposicional con función adverbial. En el Diccionario de la Lengua Española de la RAE (2014, en línea), en plan se define en un sublema del lema plan:

m. coloq. Actitud o propósito. Todo se llevó a cabo en plan amistoso.

En plan adverbial, entonces, tiene dos significados principales. El primero es 'en actitud de', ya ilustrado por la definición arriba; en estos casos, el valor semántico de la locución se acerca al uso del adverbio como. En segundo lugar, en plan significa 'a propósito de', 'con el objetivo de':

(1) Normalmente los que franquean su correspondencia y no prestan la más mínima atención a los signos postales en plan de conservarlos. (Rodríguez Abruñeiras 2015: 38)

En el Corpus Diacrónico del Español, la construcción aparece por primera vez en el año 1875, en la obra Historia crítica de la revolución española de Joaquín Costa:

(2) sino a sabiendas y reflexivamente, en plan meditado y no como obra de unos pocos.

No obstante, a pesar de algunas ocurrencias tempranas como estas, la expresión solo se generaliza a mediados del siglo xx (Nord 2006; Méndez Orense 2016).

A partir de los años setenta, en plan se ha convertido rápidamente en uno de los MMDD más frecuentemente utilizados en el lenguaje juvenil peninsular. Algunos estudios previos (Nord 2006; Jørgensen 2009; Rodríguez Abruñeiras 2015; Méndez Orense 2016) se basan en datos que provienen de tres corpus diferentes: el CREA (con datos desde 1975 hasta 2004), el COLAm (con datos de entre 2002 y 2004) y un corpus elaborado en WhatsApp en 2016 basado tanto en mensajes de texto como en archivos de audio (Méndez Orense 2016). Según estos estudios, en plan como MD cumple cinco funciones semántico-pragmáticas diferentes en el lenguaje hablado coloquial, a saber, las de MD conversacional metadiscursivo o muletilla, MD aproximativo, introductor de discurso referido, reformulador y atenuador.

En primer lugar, estudios previos sobre en plan indican que el MD funciona como muletilla o MD estructurador conversacional (tal y como ha sido definido por Cortés Rodríguez 1991 o Zorraquino y Portolés 1999) que mantiene la cohesión en el discurso y aporta a la fluidez de las interacciones: 
(3) en plan así ehhh - Y luego llega el sábado y y y - Y nada en plan pero así llevan dos meses vale (Rodríguez Abruñeiras 2015: 44)

Luego, Méndez Orense (2016: 133) afirma que en plan sirve de MD aproximativo con un valor de imprecisión, que se acerca a más o menos:

(4) Los lunes no puedo dar las clases porque está ocupado el colegio entonces tengo que darlas en plan martes o jueves o miércoles o viernes...

(5) Pues tía es justo del mismo color que un forro que tiene mi vestido en plan moradito.

Cabe indicar que ninguno de los demás estudios sobre en plan menciona esta función y, como veremos más adelante, tampoco aparece este valor en Twitter.

Una tercera función que asume en plan es la de introductor de discurso referido (Nord 2006; Rodríguez Abruñeiras 2015; Méndez Orense 2016). Para esta función se suele usar en español un verbo dicendi (por ejemplo decir, afirmar), pero actualmente se observa que en plan se ha convertido en uno de los introductores de discurso más prototípicos en el lenguaje juvenil peninsular:

(6) O sea sí me enviaron un correo me dijeron en plan noo hemos seleccionado a otro no se qué, pero... Y les he contestado en plan muchas gracias por todo y sí. (Méndez Orense 2016: 136)

Una cuarta función de en plan (Nord 2006; Rodríguez Abruñeiras 2015; Méndez Orense 2016) es la de reformulador del discurso, acercándose a otros MMDD como o sea, es decir, mejor dicho, etc. Siguiendo la tipología de los reformuladores de Zorraquino y Portolés (1999), en plan actúa en la mayoría de los casos como reformulador explicativo (tal y como o sea) que aclara lo anteriormente dicho:

(7) No sé si hablarle o no, en plan pa decirle que no le doy importancia y que tan amigos como siempre. (Méndez Orense 2016: 136)

En (7), en plan sirve para explicar a lo que refiere el hablante cuando dice hablarle. Son menos frecuentes los casos en que en plan sirve como reformulador de ejemplificación, como sinónimo de por ejemplo:

(8) Todo es más barato / en plan comida y tal / (Rodríguez Abruñeiras 2015: 41)

En plan actúa también como reformulador recapitulativo, igual que en definitiva:

(9) El nota está híper rayado diciendo un montón de mierda como que ojalá... eh... la gente con la que se junta ahora, eh... siga... con ella, que... 
que... ay, qué más, eh... que ha cambiado un montón como que ahora se ha vuelto una guarrilla que no sé qué, en plan tío un montonazo de cosas. (Méndez Orense 2016: 137)

Una última función que asume en plan en el lenguaje hablado coloquial es la de atenuador (o hedge en términos de Jørgensen 2009) (Rodríguez Abruñeiras 2015; Méndez Orense 2016). La atenuación o mitigación es «una actividad argumentativa (retórica) estratégica de minimización de la fuerza ilocutiva y del papel de los participantes en la enunciación para lograr llegar con éxito a la meta prevista» (Briz y Albelda 2013: 292) ${ }^{4}$. Una posible estrategia de atenuación consiste en el empleo de MMDD atenuantes (Cestero Mancera 2012), tal y como lo hace en plan en el ejemplo (10):

(10) A: sí es que tengo muchas camisas que me han pasado mis tíos, entonces

B: pero $u \%$ ponte una que te sienta bien, no la típica que te quede en plan a presión o la típica que te quede en plan grande (Jørgensen 2009: 14)

Aquí, el hablante utiliza en plan para mitigar el impacto de palabras negativas como a presión y grande.

\section{EN PLAN EN TWITTER}

La presente parte del estudio analiza el comportamiento funcional de en plan en la red social Twitter. El objetivo principal es comparar las funciones antedichas del MD en el lenguaje hablado coloquial (Sección 2) con las que tiene en el lenguaje en Twitter. Por ende, el análisis comparativo permite comprobar hasta qué punto el lenguaje en Twitter refleja rasgos coloquiales inherentes al registro oral.

\subsection{Metodología}

Para la elaboración del corpus se coleccionaron de manera arbitraria 300 ocurrencias de en plan en Twitter a lo largo de trece días, a saber entre el 13 y el 25 de febrero de 2019. Los ejemplos fueron recogidos a través de la máquina de búsqueda avanzada en el mismo Twitter a la que fue aplicado

${ }^{4}$ Brown y Levinson (1982) han investigado la atenuación con énfasis en el concepto de 'face', que se define como la imagen que cada persona presenta de sí misma, es decir, «the positive social value each person effectively claims for him or herself in the public arena, proffered and thus exposed throughout interaction» (Yus 2011: 272). Para evitar que durante una interacción esta imagen sea dañada, los hablantes recurren en sus actos de habla a estrategias atenuantes. 
un filtro geográfico. En concreto, solo fueron obtenidos tuits enviados desde Madrid $^{5}$. Este parámetro geográfico se justifica por pretender comparar los usos de en plan en Twitter con su uso en el corpus madrileño COLAm (véase Sección 4). Introdujimos diariamente en varios momentos la palabra clave «en plan» en la máquina de búsqueda y seleccionamos los primeros tuits en que en plan funciona como $\mathrm{MD}^{6}$. También se tomó en cuenta el perfil sociolingüístico de los hablantes, de modo que solo fueron tomados en consideración los tuits cuyo autor especifica su edad y género en la biografía de su perfil. En concreto, Rodríguez Abruñeiras (2015) y Méndez Orense (2016) afirman que en el lenguaje oral coloquial, en plan se usa sobre todo por estudiantes femeninas jóvenes. Es interesante comprobar si el mismo perfil se observa a través del uso del MD en Twitter ${ }^{7}$.

En cuanto al propio análisis, para cada caso se apuntaron la edad y el género del usuario, así como la función pragmática que asume en plan en el tuit. Para el parámetro del género distinguimos dos categorías: mujer o hombre. Para el parámetro de la edad, distinguimos tres, basándonos en Méndez Orense (2016), que afirma que los usuarios típicos de en plan son estudiantes: menos de 18 años (es decir, los estudiantes de la enseñanza secundaria), entre 18 y 25 años (los estudiantes universitarios o individuos en estrecho contacto con estudiantes universitarios) y más de 25 años (que normalmente ya no son estudiantes). Para determinar la función pragmática del MD nos hemos basado en parámetros tanto semánticos como contextuales, siguiendo, por ejemplo, el método aplicado por Enghels y Azofra Sierra (2018). Desde una perspectiva semántica, dos valores de en plan permiten sustituirse por un sinónimo que asume la misma función: en plan como introductor de discurso por una forma del verbo decir o por como y su uso como reformulador por o sea, mejor dicho o por ejemplo. Desde una perspectiva contextual, en plan intensificador casi siempre se acompaña de otros elementos que añaden un valor de intensificación (como, por ejemplo, superlativos o repeticiones) y la función atenuadora de otros elementos atenuantes (como, por ejemplo, el verbo creer, la frase que no tengo ni

Los ejemplos del corpus, por consiguiente, no provienen necesariamente todos de madrileños, sino de personas que se encontraban en ese momento en la ciudad. Sin embargo, dado que el objetivo principal del presente artículo no implica un análisis dialectal, este modo de seleccionar los datos no debe constituir un inconveniente. Cabe añadir que tanto el periodo de búsqueda como el número de 300 ocurrencias son decisiones metodológicas arbitrarias que, creemos, contribuyen a compilar un muestreo representativo del marcador.

${ }^{6}$ Esto significa que los mensajes en que en plan asume una función adverbial en vez de actuar como MD pragmático y los tuits que contienen comentarios metalingüísticos sobre el uso excesivo de en plan no fueron incorporados en el corpus.

7 Siguiendo este objetivo, las ocurrencias para las cuales no se puede obtener información sociolingüística sobre el género y la edad del usuario también han sido eliminadas del corpus final. 
$i d e a$, etc.). Cabe decir, por último, que existen casos para los cuales no es posible determinar una sola función. Jørgensen (2009: 102) afirma que "like other discourse markers, en plan is multifunctional, and can often have more than one function at the same time». Entraremos más profundamente en esta cuestión a finales de la Sección 2.2.

\subsection{Resultados}

En lo que sigue, se ofrece una descripción de las funciones discursivopragmáticas que asume en plan en Twitter. Distinguimos entre cuatro macrofunciones de en plan, a saber, como (1) introductor de discurso referido, (2) reformulador, (3) intensificador y (4) atenuador. Además, dos de estas macrofunciones engloban diversas microfunciones: la función de introductor de discurso se subdivide en introductor de discursos hablados o escritos, de pensamientos y de sentimientos, mientras que en plan reformulador se subdivide en reformulador explicativo, rectificativo y de ejemplificación. Finalmente, observamos que estas funciones pueden acumularse dentro de una misma ocurrencia. La tabla 1 resume las frecuencias de estas funciones en el corpus.

TABLa 1. Distribución de las funciones discursivo-pragmáticas de en plan en Twitter

\begin{tabular}{lcc}
\multicolumn{1}{c}{ Función } & \# absoluto & \# relativo \\
\hline Introductor de discurso & 159 & $53 \%$ \\
Reformulador & 92 & $30,7 \%$ \\
Intensificador & 21 & $7 \%$ \\
Atenuador & 19 & $6,3 \%$ \\
Múltiples funciones & 9 & $3 \%$ \\
\hline TOTAL & 300 & $100 \%$ \\
\hline
\end{tabular}

En el corpus de tuits elaborado, en plan se ha especializado pragmáticamente, ya que la macrofunción de introductor de discurso referido predomina y constituye el $53 \%$ de los casos $(n=159)$. Existe en el corpus una enorme variedad en cuanto a la naturaleza del discurso referido, por lo que se distinguen varias microfunciones (ver también Palacios Martínez 2014). En concreto, en plan puede introducir un discurso escrito (11) o hablado (12): 
(11) La gente que escribe su móvil en la puerta de los aseos en plan: «quiero sexo. Busco tío activo. Llámame y follamos». ¿De verdad esperan que les llamen? $(13 / 02 / 2019)$

(12) Es que además se ha ofendido porque me ha dicho pa cuando concertamos una cita ay yo en plan estamos aún en proceso de selección $(21 / 02 / 2019)$

Además, en plan también introduce pensamientos (13), tanto de los hablantes mismos como de otros:

(13) Por eso pregunto! Yo no tengo truco, me lo aprendí de memoria y ya está, no hago en plan $8+2=10 / 6-2=4$ entonces 14 ! (24/02/2019)

Por último, el MD sirve para introducir emociones y sentimientos, expresados mediante palabras escritas, emoticonos (14), fotos, gifs y signos de puntuación (15):

(14) Al fin le hablé y su respuesta fue igualmente jajaja Me quedado en plan (19/02/2019)

(15) Está mirando a Karol en plan ??. (13/02/2019)

La segunda función más frecuente (el 30,7\%, n = 92) de en plan en el corpus es la de reformulador. El MD funciona como reformulador explicativo en la mayoría de los casos $($ el 84,8\% $(n=78)$ de las ocurrencias de en plan reformulador), tal y como o sea, es decir:

(16) Mi hermana se ha ido a la playa y tengo que curar al gato en plan limpiarle la cicatriz yo sola prfffvrrrr que alguien me ayudeeeee (23/02/2019)

El MD también sirve de reformulador rectificativo, acercándose a más bien o mejor dicho, en el 9,8\% $(\mathrm{n}=9)$ de los casos $(17)$. Es interesante señalar que esta función no se incluye en la mayoría de los estudios previos sobre su funcionamiento en el lenguaje coloquial.

(17) Me apetece subir una foto a instagram pero no tengo una foto buena, en plan, tengo del viaje a Moscú pero no quiero subir más de ese viaje por no saturar $(20 / 02 / 2019)$

Otra función de en plan como reformulador es la de ejemplificación, siendo un sinónimo de por ejemplo o como, que ocurre en el 5,4\% (n=5) de los casos:

(18) voy por épocas, pero ahora me flipan los colores cálidos en plan naranja, rojo etc. $(19 / 02 / 2019)$

En tercer lugar, cuando sirve para subrayar determinado aspecto de lo dicho, en plan asume una función intensificadora en el $7 \%$ de los casos 
( $n=21)(19-20)$. Destacamos que esta función se menciona en el artículo de Pons Rodríguez publicado en El País: «Estamos en plan explicando la expresión en plan» (2018), pero no se incluye en ningún otro estudio previo.

(19) Es que si nos hace un Work your magic o Shady Lady 2 es en plan... WOW $(16 / 02 / 2019)$

(20) Bueno, mi hazaña de hoy ha sido cortarme el dedo en plan MUCHO mientras cortaba papeles $\odot(19 / 02 / 2019)$

Para continuar, la función atenuante de en plan constituye el $6 \%(\mathrm{n}=19)$ de los casos en el corpus. Se destaca su uso como estrategia para mitigar insultos (21) y enunciados sobre cosas incómodas o desagradables como la pornografía infantil $(22)^{8}$.

(21) el ronal es como tontito no en plan? (21/02/2019)

(22) Pa empezar es que no entiendo en qué cabeza puede parecer bien que te gusten los dibujitos de pornografía infantil. En plan por muy dibujitos que sean SIGUE SIENDO PUTA PORNOGRAFÍA INFANTIL AUNQUE NO ESTÉS EXPLOTANDO SEXUALMENTE A UN CRÍO ESTÁS PAJEANDOTE PENSANDO EN UNO?? (20/02/2019)

Por último, el muestreo incluye 9 tuits en que diferentes funciones discursivo-pragmáticas de en plan se acumulan:

(23) Sobre todo el óscar a la mejor puta fotografía y el de edición en plan wtf, y los de vestuario me dan mazo pena, y hasta un puto corto van a quitar, es que no me jodas $(14 / 02 / 2019)$

En (23), en plan primero introduce un pensamiento (wtf) del hablante al ver las nominaciones a los óscar. Segundo, en plan también sirve de intensificador subrayando su sorpresa, tal y como otros elementos intensificadores en el tuit como puta/puto y mazo.

\section{RASGOS DE LA ORALIDAD COLOQUiAL EN TWITTER}

Después de haber indicado las funciones que cumple en plan en Twitter hoy día, resulta interesante la comparación con el uso del MD en el lenguaje hablado. Para este propósito, nos basamos en los trabajos de Nord (2006) y Rodríguez Abruñeiras (2015), que han investigado en plan en el corpus

${ }^{8}$ La función atenuante es fácilmente detectable por la presencia de otros elementos atenuantes en los tuits: en los ejemplos aquí citados, llama la atención el uso del diminutivo (tontito, dibujitos). 
oral COLAm ${ }^{9}$; sus resultados se resumen en la tabla 2. Se observan ya algunas diferencias cuantitativas entre ellos, que pueden explicarse por la distancia cronológica (nueve años) entre el estudio de Nord y el de Rodríguez Abruñeiras y, eventualmente, por la extensión de sus respectivos corpus (173 ocurrencias de en plan en el estudio de Nord y 410 en el análisis de Rodríguez Abruñeiras).

TABLA 2. Funciones de en plan en el corpus COLAm y Twitter (2019)

\begin{tabular}{lccc}
\hline \multicolumn{1}{c}{ Función de en plan } & $\begin{array}{c}\text { COLAm } \\
\text { (Nord 2006) }\end{array}$ & $\begin{array}{c}\text { (Rodríguez Abruneiras } \\
\text { 2015) }\end{array}$ & $\begin{array}{c}\text { Twitter } \\
\mathbf{( 2 0 1 9 )}\end{array}$ \\
\hline Muletilla & $60 \%$ & $75 \%$ & $/ /$ \\
Introductor de discurso & $25 \%$ & $20 \%$ & $53 \%$ \\
\hline Reformulador & $15 \%$ & $5 \%$ & $30,7 \%$ \\
Intensificador & $/ /$ & $/ /$ & $7 \%$ \\
Atenuador & $/ /$ & $/ /$ & $6,3 \%$ \\
Multifuncional & $/ /$ & $/ /$ & $3 \%$ \\
\hline TOTAL & $100 \%$ & $100 \%$ & $100 \%$ \\
\hline
\end{tabular}

Antes de pasar al análisis funcional, resulta interesante comprobar en qué medida es similar el perfil de los usuarios de en plan en los corpus orales y Twitter. Los estudios basados en el COLAm llegan a la conclusión de que en plan es utilizado sobre todo por mujeres jóvenes. Nuestro corpus basado en Twitter confirma este dato. En cuanto al parámetro del género, el 67,7\% ( $\mathrm{n}=203)$ de las ocurrencias de en plan provienen de mujeres, el $32,3 \%(n=97)$ de hombres. Puesto que los hombres españoles son mayoritarios en Twitter (el 54\% de los usuarios) (<MásQueNegocio.com>, septiembre 2019), es reforzada aún más la hipótesis de que las mujeres tienden a utilizar en plan más frecuentemente. En cuanto al parámetro de la edad, el $84,5 \%(n=256)$ de las ocurrencias provienen de estudiantes universitarios de entre 18 y 25 años. Esta tendencia resulta aún más llamativa si tomamos en cuenta que los individuos de entre 16 y 24 años solo constituyen un $21 \%$ de los usuarios españoles en Twitter.

9 La comparación con el corpus oral CREA no resulta interesante ya que solo contiene 26 ocurrencias de en plan como MD (Rodríguez Abruñeiras 2015). Tampoco resulta posible una comparación con el corpus elaborado a través de WhatsApp por Méndez Orense (2016), ya que esta autora analiza tanto mensajes de texto como archivos de audio sin indicar frecuencias de los valores de en plan en las dos variantes distintas. 
Si comparamos en plan en Twitter con en plan en el corpus COLAm desde una perspectiva funcional, tres diferencias principales llaman la atención:

1) La función de muletilla de en plan queda ausente en Twitter, mientras que en el COLAm se observa en la gran mayoría de los casos.

2) En Twitter, las funciones de introductor de discurso y reformulador predominan ampliamente (el 83,7\%), mientras que en el COLAm estas funciones no son tan frecuentes (aproximadamente un cuarto de los casos).

3) En Twitter surge una función de en plan que no está presente en los corpus orales, a saber, la de intensificador.

Teniendo en cuenta estas diferencias, nuestra hipótesis será que, por una parte, el lenguaje en Twitter se distancia del lenguaje oral coloquial prototípico por la ausencia del cambio de turnos. Por otra parte, los hablantes en Twitter adoptan un estilo «involucrado» y «afectado» (involved) que se considera propio de la oralidad y la coloquialidad (Chafe 1982; Biber 1988).

En primer lugar, se observa que en plan no se utiliza como muletilla en Twitter. Esto no sorprende si tomamos en consideración el carácter particular de la conversación en la red social (Bruns y Burgess 2011; Rossi y Magnani 2012). Prototípicamente, los tuits son intervenciones en que un hablante se dirige a un grupo amplio de personas (sus seguidores) sin esperar una respuesta. La interacción, entonces, es sobre todo monológica. Otros usuarios pueden responder a los tuits, pero en estos casos la respuesta llega más tarde, por lo que la interacción es asincrónica. En Twitter falta, en otras palabras, el cambio de turnos típico de la conversación oral cara a cara, por lo que resulta innecesario un MD que «rellena silencios en la conversación» e «indica cambios de turno» (Nord 2006: 30).

En segundo lugar, se nota que los hablantes en Twitter adoptan un estilo «involucrado», es decir, expresivo y animado, que implica al interlocutor en su discurso. Chafe (1982) y Biber (1988) consideran el binario involvement (involucramiento) vs. detachment (distanciamiento) como uno de los rasgos más útiles para distinguir entre respectivamente el lenguaje hablado y escrito (ver también Koch y Oesterreicher 2007). El lenguaje hablado se caracteriza por involvement, por la expresividad y el involucramiento de los hablantes en la conversación: «Involvement refers to those linguistic features which reflect the fact that speaker and listener typically interact with one another, while writer and reader typically do not» (Biber 1988: 43). Adoptar un estilo involucrado, entonces, significa tomar conciencia del interlocutor. Varios mecanismos lingüísticos contribuyen a este estilo (Chafe 1982; Collot y Belmore 1996): (a) el discurso directo; (b) partículas enfáticas; (c) el uso creativo de elementos paralingüísticos como la entonación, el volumen, la mímica; (d) la repetición, y (e) la atenuación. Si analizamos 
el corpus teniendo en la mente estos rasgos, concluimos que el lenguaje en Twitter es muy expresivo y animado.

De hecho, resulta notable la alta frecuencia (el $53 \%[\mathrm{n}=159])$ de la función de introductor de discurso referido de en plan. Como hemos indicado en el apartado 2.2, esta función del MD le permite al hablante representar discursos, pensamientos y emociones del hablante mismo o de otros, de modo que la conversación se vuelva más animada. Una segunda estrategia utilizada para alcanzar un estilo expresivo consiste en el empleo de elementos enfáticos. Aquí resulta llamativa la presencia en el corpus de una función no mencionada en estudios previos, a saber, la de en plan como intensificador. En tercer lugar, el rasgo del uso creativo de elementos paralingüísticos se traslada a Twitter por la ortografía. De hecho, los usuarios utilizan signos de puntuación, mayúsculas y emoticonos para indicar cambios de entonación, señalar diferencias de volumen, etc., como ilustran los casos (24-25):

(24) Gente que te habla solo con audios y tú estás en plan, BITCH estoy escuchando música en serio crees que la voy a parar para oírte??????????

$(14 / 02 / 2019)$

(25) $\mathrm{Al}$ fin le hable y su respuesta fue igualmente jajaja. Me quedado en plan (1) $(17 / 02 / 2019)$

En (24), el hablante utiliza mayúsculas para indicar que está gritando; en (25) utiliza un emoticono para imitar su expresión facial al oír algo. Una cuarta estrategia para generar más expresividad es a través de la repetición. De hecho, en plan actúa frecuentemente (en el 30,7\% [n = 92] de los casos) como reformulador explicativo, introduciendo la repetición de un discurso. Ciabarri (2013) confirma que la reformulación explicativa se relaciona más con el lenguaje oral, pues se dirige explícitamente hacia el oyente: asume una función didáctica y ayuda al oyente a interpretar una posible dificultad en el texto. En (26), por ejemplo, el hablante utiliza en plan para explicar lo que significa exactamente curar al gato:

(26) Mi hermana se ha ido a la playa y tengo que curar al gato en plan limpiarle la cicatriz yo sola prfffvrrrr que alguien me ayudeeeee (23/02/2019)

Además, en los tuits en que en plan asume otra función, también observamos mucha repetición. En (27), por ejemplo, en plan sirve como intensificador e introduce una repetición de moñas:

(27) ayer estaba que queria tirar a alguien por la ventana, hoy estoy moñas pero en plan moñas $(19 / 02 / 2019)$

Por último, la presencia de la función atenuante de en plan en Twitter también demuestra que los usuarios toman conciencia de la presencia de 
sus interlocutores. De hecho, si se tiene en cuenta que en Twitter un hablante se dirige a un grupo muy amplio de oyentes (a saber, los seguidores de un usuario) que no están presentes físicamente, y a veces anónimos, puede resultar paradójica la presencia de la atenuación en la red social. La presencia de la atenuación en Twitter, entonces, demuestra que en la mente del autor, los demás usuarios no están tan despersonalizados y el discurso se acerca a una conversación cara a cara.

Cabe mencionar que existe la posibilidad de que el estilo expresivo del lenguaje en Twitter no se produzca para imitar la oralidad, sino que se deba al perfil sociolingüístico de los hablantes analizados. Jørgensen (2009) y Méndez Orense (2016) afirman que la lengua de mujeres jóvenes se define prototípicamente como expresiva (Herring 1996; Andersen 2001). Sin embargo, el corpus analizado parece refutar estas ideas estereotipadas. Consideremos el ejemplo (28), que proviene de un hombre mayor de 25 años:

(28) Buah, te entiendo tantísimo. Yo estoy en plan «joder no soy capaz de moverme vivo cansado y triste» y luego caigo en la época y el tiempo y -_$(21 / 02 / 2019)$

Observamos diferentes recursos para generar un estilo involucrado, como por ejemplo el uso creativo de los signos de puntuación (el emoticono -__ para expresar el aburrimiento), la intensificación (tantísimo), el uso del discurso directo, etc.

Para concluir, comparando el comportamiento funcional de en plan en el corpus COLAm y el corpus con base en Twitter, se observa alguna ambivalencia. Por una parte, por el carácter monológico y asincrónico de los tuits, el lenguaje en Twitter se distancia del lenguaje hablado coloquial, ya que falta el cambio de turnos. Por consiguiente, la función de muletilla de en plan (que sirve, entre otras cosas, para organizar el cambio de turnos) queda ausente en la red social. Por otra parte, el lenguaje en Twitter se acerca bastante a la oralidad coloquial por el estilo muy involucrado y expresivo que adoptan los usuarios. La búsqueda de este estilo involucrado en Twitter explica la alta frecuencia de las funciones de introductor de discurso referido y reformulador (explicativo) en comparación con el corpus oral y el surgimiento de sus nuevas funciones de intensificador y atenuador.

\section{Conclusiones}

Para concluir, este estudio ha contribuido a la discusión sobre el lugar que ocupa el lenguaje utilizado en Twitter en el continuum de la variación conceptual o inmediatez comunicativa de diferentes géneros discursivos (Koch y Oesterreicher 2007). El objetivo principal ha sido comprobar en 
qué medida esta variante refleja el registro hablado coloquial y si es apta para ser utilizada como corpus lingüístico oral. Para tal propósito, pasamos al estudio de caso de en plan como MD en el español peninsular. El análisis se ha centrado en tres preguntas de investigación:

1) ¿Cuáles son las funciones discursivo-pragmáticas que asume el MD en plan hoy día en la red social Twitter?

2) ¿En qué medida se comparan las funciones de en plan en Twitter con las que asume el MD en el lenguaje hablado coloquial?

3) ¿Puede considerarse el lenguaje utilizado en Twitter un reflejo del registro oral coloquial?

A base de un corpus elaborado en Twitter, concluimos que en plan asume cuatro funciones discursivo-pragmáticas principales: introductor de discurso referido, reformulador (tanto explicativo como rectificativo y de ejemplificación), intensificador y atenuador. Además, estas funciones pueden acumularse como ocurrencia del MD. Comparando estas conclusiones con los resultados de estudios previos del lenguaje hablado en el corpus COLAm, se destacan tres diferencias principales: primero, la función de muletilla de en plan queda ausente en Twitter, mientras que en el COLAm se observa en más del $50 \%$ de los casos; segundo, en Twitter las funciones de introductor de discurso y reformulador constituyen la gran mayoría de los casos (el $83,7 \%$ ), mientras que en el COLAm estas funciones se presentan con una frecuencia mucho más baja; tercero, en Twitter surge una función de en plan que no está presente en los corpus orales, a saber, la de intensificador.

Teniendo en cuenta estas diferencias, concluimos que se necesita cierta precaución si se decide usar Twitter como corpus lingüístico que refleja el registro oral coloquial. De hecho, por una parte en Twitter se pierde un rasgo fundamental de la oralidad: los tuits, por su carácter monológico y asincrónico, no se caracterizan por el cambio de turnos propio del registro oral, lo que explica por qué queda ausente la función de muletilla en Twitter. Para la investigación sobre elementos lingüísticos condicionados por el cambio de turnos (como por ejemplo el vocativo y los MMDD conversacionales), Twitter no resulta tan apto como corpus. Por otra parte, en Twitter los hablantes adoptan un estilo involucrado, expresivo y animado, propio del registro coloquial, al que se llega mediante diferentes mecanismos, como la incorporación del discurso directo y de partículas enfáticas, el uso creativo de signos de puntuación, emoticonos y mayúsculas, la repetición, la reformulación explicativa y la atenuación. A este respecto, Twitter sí imita al registro oral coloquial y, como consecuencia, para la investigación sobre estos elementos lingüísticos ligados a la expresividad resulta más adecuado como corpus. 


\section{BIBLIOGRAFÍA}

ANDERSEN, Gisle (2001): Pragmatic Markers and Sociolinguistic Variation: A Relevancetheoretic Approach to the Language of Adolescents, Ámsterdam/Filadelfia: John Benjamins Publishing Company.

BIBER, Douglas (1988): Variation across Speech and Writing, Nueva York: Cambridge University Press.

BRIZ, Antonio (1998): El español coloquial en la conversación. Esbozo de pragmagramática, Barcelona: Ariel.

- (2003): «La interacción entre jóvenes. Español coloquial, argot y lenguaje juvenil». En Maria Teresa Echenique Elizondo y Juan Sánchez Sánchez Méndez (eds.), Lexicografía y lexicología en Europa y América, Madrid: Gredos, 141-154.

- (2012): «Los déficits de los corpus orales del español (y de algunos análisis)». En Tomás Jiménez Júlia et al. (eds.), Cum corde et in nova gramatica, Santiago de Compostela: Universidade de Santiago de Compostela, 115-137.

- (2014): «Hablar electrónicamente por escrito», Chimera: Romance corpora and linguistic studies 1, 77-89.

BRIZ, Antonio y Marta Albelda (2013): «Una propuesta teórica y metodológica para el análisis de la atenuación lingüística en español y portugués. La base de un proyecto en común (ES.POR.ATENUACIÓN)», Onomázein 28, 288-319.

Bruns, Axel y Jean E. Burgess (2011): «\#Ausvotes: how Twitter covered the 2010 Australian Federal Election», Communication, Politics and Culture 44/2, 37-56.

Cestero Mancera, Ana María (2012): «Recursos lingüísticos de atenuación en el habla de Madrid. Estudio sociopragmático». En Tomás Jiménez Júlia et al. (eds.), Cum corde et in nova gramatica, Santiago de Compostela: Universidade de Santiago de Compostela, 233-246.

Chafe, Wallace (1982): «Integration and Involvement in Speaking, Writing and Oral Literature». En Deborah Tannen (ed.), Spoken and Written Language: Exploring Orality and Literacy, Nueva Jersey: ABLEX Publishing Corporation, 35-54.

CIABARRI, Frederica (2013): «Italian Reformulation Markers: A Study on Spoken and Written Language». En Catherine Bolly y Liesbeth Degand (eds.), Across the Line of Speech and Writing Variation. Corpora and Language in Use - Proceedings 2, Louvain-la-Neuve: Presses universitaires de Louvain, 113-127.

CLAVE (Diccionario de uso del español actual) (1997): «plan», Madrid: SM, 1431.

Collot, Milena y Nancy Belmore (1996): «Electronic language: a new variety of English». En Susan Herring (ed.), Computer-Mediated Communication: Linguistic, Social and Cross-cultural Perspectives, Ámsterdam/Fildelfia: John Benjamins Publishing Company, 13-28.

Cortés RodríGuez, Luis (1991): Sobre conectores, expletivos y muletillas en el español hablado, Málaga: Ágora. 
CRYstal, David (2001): Language and the Internet, Cambridge: Cambridge University Press.

DE SMET, Emma (2019): ¿Nuevo marcador o moda pasajera? La gramaticalización del marcador discursivo en plan: un análisis lingüistico en Twitter. Tesis inédita de la Universiteit Gent.

Enghels, Renata y Elena Azofra Sierra (2018): «Sobre la naturaleza de los corpus y la comparabilidad de resultados en lingüística histórica: Estudio de caso del MD pragmático sabes», Spanish in Context 15/3, 465-489.

Fuentes, Catalina (1996): La sintaxis de los relacionantes supraoracionales, Madrid: Arco/Libros.

GILQuin, Gaëtanelle y Sylvie DE CocK (2011): «Errors and Disfluencies in Spoken Corpora: Setting the Scene», International Journal of Corpus Linguistics 16/2, 141-172.

GONZÁLEZ FERnÁNDEZ, Adela (2017): «Estudio de neologismos a través de big data en un corpus textual extraído de Twitter», ELUA. Estudios de Lingüística 31, 171-186.

Herring, Susan (1996): «Two Variants of an Electronic Message Schema». En S. Herring (ed.), Computer-mediated Communication: Linguistic, Social, and Crosscultural Perspectives, Ámsterdam/Filadelfia: John Benjamins Publishing Company, 81-108.

- (2004): «Computer-mediated Discourse Analysis: An Approach to Researching Online Behavior». En Sasha A. Barab et al. (eds.), Designing for Virtual Communities in the Service of Learning, Nueva York: Cambridge University Press, 338376.

JøRGENSEN, Annette Myre (2009): «En plan used as a Hedge in Spanish Teenage Language». En Anna-Brita Stenström y Annette Myre Jørgensen (eds.), Youngspeak in a Multilingual Perspective, Ámsterdam/Filadelfia: John Benjamins Publishing Company, 95-118.

Koch, Peter y Wulf Oesterreicher (2007): Lengua hablada en la Romania: español, francés, italiano, Madrid: Gredos.

LóPEz Serena, Araceli y Margarita Borreguero ZulOAga (2010): «Los marcadores del discurso y la variación lengua hablada vs. lengua escrita». En Óscar Loureda Lamas y Esperanza Acín-Villa (coords.), Los estudios sobre MDes del discurso en español, hoy, Madrid: Arco/Libros, 415-493.

MásQueNegocio.com (2016): «¿Cómo es el usuario de Twitter en España?». <https://www.masquenegocio.com/2016/01/27/usuario-twitter-espana>.

MÉndez Orense, María (2016): «Valores pragmático-discursivos de la construcción lingüística en plan. ¿Formación de un nuevo MD?», Philología Hispalensis 30/1-2, 123-144.

Moliner, María (1998): «plan», Diccionario de uso del español, Madrid: Gredos, 696.

Nishimura, Yukiko (2013): «A Stylistic Continuum of Speech, CMC and Writing: A Comparative Linguistic Analysis of Japanese Texts». En Catherine Bolly y Liesbeth Degand (eds.), Across the Line of Speech and Writing Variation. Corpora and Language in Use - Proceedings 2, Louvain-la-Neuve: Presses universitaires de Louvain, 113-127. 
NorD, Magni (2006): En plan en plan científico. Las funciones de en plan en el lenguaje juvenil de Madrid: estudio descriptivo. Tesis inédita de la Bergen University.

Overbeck, Anja (2017): «Orality and Literacy of Online Communication». En Kristina Bedijs y C. Maaß (eds.), Manual of Romance Languages in the Media, Berlín: De Gruyter, 176-202.

Padilla Herrada, María Soledad (2016): «Marcadores y partículas discursivas interactivas en el entorno político/periodístico de Twitter», Philologia Hispalensis 30/1, 193-212.

Palacios Martínez, Ignacio Miguel (2014): «The Quotative System in Spanish and English Youth Talk. A Contrastive Corpus-based Study», Miscelánea 49, 95-114.

PAno Alamán, Ana (2015): «Aproximación a los MDes del discurso en los comentarios de la prensa digital española y argentina», RILL 20, 89-114.

- y Ana MANCERA Rueda (2014): «La "conversación” en Twitter: las unidades discursivas y el uso de MDes interactivos en los intercambios con parlamentarios españoles en esta red social», Estudios de Lingüística del Español 35/1, 234-268.

Pons RodríGUEZ, Lola (2018): «Estamos en plan explicando la expresión en plan», El País, 2 de abril 2018. <https://verne.elpais.com/verne/2018/04/01/articu lo/1522599285_066782.html>.

PorTOLÉs, José (1998): Marcadores del discurso, Barcelona: Ariel.

Real Academia Española (2014): Diccionario de la Lengua Española [en línea], 23. edición, Madrid: Espasa Calpe. <https://dle.rae.es/?w=>.

RECALDE, Montserrat y Victoria VÁzQuez Rosas (2009): «Problemas metodológicos en la formación de corpus orales». En Pascual Cantos Cómez y Aquilino Sánchez Pérez (eds.), A Survey of Corpus-based Research. Murcia: Asociación Española de Lingüística del Corpus, 51-64. <https://www.um.es/lacell/aelinco/ contenido/pdf/4.pdf $>$.

Rodríguez AbruñeIRAS, Paula (2015): De función adverbial a MD del discurso: Origen, gramaticalización y uso actual de «En Plan (de)» en el español peninsular. Tesis inédita de la University of Wisconsin-Milwaukee.

Rossi, Luca y Matteo Magnani (2012): «Conversation Practices and Network Structure in Twitter», Proceedings of the Sixth International AAAI Conference on Weblogs and Social Media. <https://www.aaai.org/ocs/index.php/ICWSM/ICWSM12/ paper/view/4634/5058>.

Shanmuganathan, Thilagavathi (2005): «Ethics and the Observer's Paradox», RELC Journal 36/1, 73-84.

Yus, Francisco (2001): Ciberpragmática. El uso del lenguaje en Internet, Barcelona: Ariel.

- (2011): «Politeness on the Net», Cyberpragmatics: Internet-mediated communication in context, Ámsterdam/Filadelfia: John Benjamins Publishing Company, 255-286.

ZorraQuino, María Antonia y José Portolés (1999): «Los marcadores del discurso». En Ignacio Bosque y Violeta Demonte (eds.), Gramática descriptiva de la lengua española, vol. III, Madrid: Espasa Calpe, 4051-4213. 